DOI: $10.35643 /$ Info.25.1.6

Artículo Original

\title{
El derecho de autor en el entorno web: prácticas y servicios bibliotecarios bajo las limitaciones y excepciones de la ley, el caso uruguayo 1
}

\section{Copyright in the web environment: library practices and services under the limitations and exceptions of the law, the Uruguayan case}

\section{Djamila Romani Parada ${ }^{a}$}

\footnotetext{
${ }^{a}$ Profesora Adjunta del Departamento de Tratamiento y Transferencia de la Información. Facultad de Información y Comunicación (FIC) Universidad de la República (UdelaR). Uruguay. Correo electrónico: djamila.romani@fic.edu.uy ORCID: 0000-0001-9609-6833
}

\section{Resumen}

El avance tecnológico posibilita el acceso masivo y remoto a la información en soporte digital. Este hecho determina el tipo de necesidades y demandas de los usuarios. La precepción de éstas por parte de los profesionales de la información define a su vez las prácticas que se instituyen y los servicios que se brindan en las bibliotecas. El marco legal vigente no autoriza muchas de las prácticas usuales que garantizan el acceso a la información. La ley de derecho de autor en Uruguay no incluye excepciones en favor de las bibliotecas.

En el presente trabajo se analizarán desde el contexto de la Sociedad de la Información las prácticas llevadas a cabo en las bibliotecas, vinculadas a la reproducción bajo el paradigma social en Ciencia de la Información.

A través de la percepción de los/as bibliotecológos/as se analizarán las dificultades concretas que impiden satisfacer las necesidades de los usuarios en el marco de la normativa vigente.

Se indagará además si Uruguay cuenta con una ley de posible cumplimiento, y los alcances y problemas que su vigencia implica para las bibliotecas.

\footnotetext{
${ }^{1}$ Síntesis de la Tesis de Maestría en Información y Comunicación defendida en el año 2019. Facultad de Información y Comunicación - Universidad de la Repúbica (FIC-UdelaR)
} 
Este trabajo pretende ser un aporte desde la Ciencia de la Información al Derecho para que el marco normativo sea de utilidad y tenga una base real de aplicación en un paradigma en el cual los usuarios se comporten informacionalmente acorde a las posibilidades que la realidad les ofrece.

Palabras clave: DERECHO DE AUTOR; LIMITACIONES Y EXCEPCIONES PARA LAS BIBLIOTECAS; ACCESO A LA INFORMACION.

\begin{abstract}
The technological advance allows the massive and remote access to the information in digital support. This fact determines the type of needs and demands of the users. The perception of these by the information professionals defines in turn the practices that are instituted and the services that are provided in the libraries. The current legal framework does not authorize many of the usual practices that guarantee access to information. The copyright law in Uruguay does not include exceptions in favor of libraries.

In the present work, the practices carried out in libraries, linked to reproduction under the social paradigm in Information Science, will be analyzed from the context of the Information Society.

Through the perception of the librarian / as will analyze the specific difficulties that prevent meet the needs of users in the framework of current regulations.

It will also be investigated if Uruguay has a law of possible compliance, and the scope and problems that its validity implies for libraries.

This work aims to be a contribution from Information Science to Law so that the regulatory framework is useful and has a real application base in a paradigm in which users behave informationally according to the possibilities that reality offers them.
\end{abstract}

\title{
Keywords: COPYRIGHT; LIMITATIONS AND EXCEPTIONS FOR LIBRARIES; ACCESS TO INFORMATION.
}

Fecha de recibido: 06/04/2020

Fecha de aceptado: 25/04/2020 


\section{Introducción}

La ley uruguaya de derecho de autor «protege el derecho moral del autor de toda creación literaria, científica o artística y le reconoce derecho de dominio sobre las producciones de su pensamiento, ciencia o arte, con sujeción a lo que establece el derecho común y los artículos siguientes». (Ley 9.739, 1937)

Como plantean Fernández-Molina y Guimarães (2018:124), las funciones primordiales de una biblioteca están ligadas a las prácticas habituales de reproducción (escaneo, fotocopia, envío de enlaces); puesta a disposición (acceso al público) y difusión de las obras.

La masiva utilización de las herramientas tecnológicas no sólo ha brindado mayores posibilidades de reproducción y acceso remoto a las obras. También inauguró nuevos usos y costumbres en cuanto a las necesidades y expectativas que tienen los usuarios en torno al acceso y la transferencia de información.

Una interpretación estricta de la ley de derecho de autor en Uruguay incluye el préstamo de libros como un acto ilícito. Advierte Díaz Charquero al respecto: «existe la amenaza de demanda a las bibliotecas exigiendo el pago de una compensación o canon por libro prestado» (Díaz Chaquero, s.f.:9). Se trata de una situación que interpela la función más primaria de las bibliotecas, como es el préstamo de documentos en soporte papel.

Existe un recurso legal propuesto en el Tratado de la OMPI sobre Derecho de Autor (1996) acordado en Ginebra: Limitaciones y Excepciones, cuyo objetivo es establecer un equilibrio entre los derechos de los autores y el derecho de acceso a información que tiene la sociedad.

En el marco del problema planteado en dicho estudio, Uruguay se encuentra en franca desventaja respecto a otros países. La legislación uruguaya no contempla limitación ni excepción en favor de las bibliotecas, tal como propone desde la Organización Mundial de la Propiedad Intelectual (en adelante OMPI) Crews, y lo mismo ocurre con las copias para uso privado o con fines de conservación.

Determinado el problema, fue configurada la siguiente hipótesis para el desarrollo de la investigación: la ley vigente sobre derechos de autor no brinda el marco legal adecuado para que las bibliotecas cumplan con las demandas y expectativas de los usuarios, que en la actualidad están directamente vinculadas a servicios 
basados en las TICS. Para ello, las prácticas de los profesionales deben orientarse a sortear el marco legal de la forma menos expuesta.

\section{Objetivos}

\subsection{Objetivo general}

Analizar en detalle y evaluar la situación respecto a las restricciones y conflictos que implica cumplir con la ley de derecho de autor vigente en Uruguay en las prácticas y servicios en las bibliotecas con vistas a realizar acciones acordes a las necesidades de los usuarios.

\subsection{Objetivos específicos}

a) Describir las prácticas bibliotecarias vinculadas a la reproducción y difusión de las obras, las posibilidades tecnológicas de acceso a la información; las demandas y necesidades de los usuarios.

b) Indagar si es posible brindar servicios acordes a las necesidades de los usuarios en conformidad con la ley vigente.

c) Consultar sobre las acciones concretas que llevan a cabo los bibliotecólogos/as ante las limitantes que plantea la ley.

d) Vehiculizar la comunicación entre las áreas del Derecho y la Ciencia de la Información y brindar una herramienta desde la bibliotecología para futuros estudios sobre modificaciones de la legislación de derecho de autor.

\section{Marco Teórico}

\subsection{El derecho de autor y las limitaciones y excepciones}

El derecho de autor, según definición del Ministerio de Educación y Cultura (MEC) de 2014, «es el sistema creado a fin de proteger las obras literarias, científicas y artísticas que surgen como fruto del intelecto del hombre». El marco normativo que aplica al derecho de autor en Uruguay se clasifica, según su naturaleza, en derechos morales y derechos patrimoniales (o de explotación) Los primeros son intransferibles, mientras que los segundos pueden ser cedidos mediante acuerdo de partes. 
El marco jurídico que rige a nivel nacional para la regulación del derecho de autor es la « Ley sobre propiedad intelectual y artística» número 9.739 del 17 de diciembre de 1937. El 10 de enero de 2003 se le introducen modificaciones través de la Ley 17.616, «Ley de derechos de autor y derechos conexos». En 26 de agosto de 2004 se aprueba la Ley 17.805, la cual modifica los artículos 22 y 24 de la Ley 17.616 (Balsa y Bugallo, 2011: 31). En su base jurídica la Ley 9.739 de Derecho de Autor en Uruguay, es ampliamente protectora del autor y de sus derechos morales y patrimoniales. Considera ilícita toda copia, reproducción y puesta a disposición de las obras sin consentimiento del autor. Data del año 1937, y sufrió modificaciones en 2003 mediante la Ley 17.616, cuando pasó a llamarse Ley de Derecho de Autor y Derechos Conexos, e incorporó otras figuras pasibles de derecho, como autores, intérpretes y/o ejecutantes.

Las limitaciones y excepciones refieren al necesario mecanismo jurídico que equilibra dos derechos: los patrimoniales y morales de los autores y conexos, y a la vez asegura el acceso de la ciudadanía a la información. Valdes Otero (1953) citado por Bugallo (2006:725), afirma: «Los derechos de autor se encuentran sometidos a restricciones destinadas a conciliar el interés individual del creador intelectual con el legítimo interés colectivo de la sociedad en cuyo seno actúa.» «El derecho de autor a la explotación de su obra lo enfrenta a la sociedad en general» (Bugallo, 2006:725) ya que la sociedad se encuentra interesada en poder acceder a las obras. «Una de las formas de equilibrar estas dos posiciones es la consagración de límites a las facultades de explotación.». (Bugallo, 2006:725)

Es por este motivo que muchos países incorporan diversos tipos de limitaciones y excepciones. $^{2}$

Díaz Charquero (s.f.:3) se refiere así a las limitaciones y excepciones: «Este sistema oficia como contrapeso jurídico habilitando el derecho de la sociedad toda al disfrute de los bienes culturales, y también colabora con la efectivización de los derechos a la educación y libertad de expresión». Por tanto, es un recurso jurídico que cobra especial relevancia en el entorno de la Sociedad de la Información y la utilización de las herramientas vinculadas a las TICS.

\footnotetext{
${ }^{2}$ En cuanto a la terminología, la literatura jurídica utiliza también: " exenciones" o " limitaciones de los derechos de los titulares del derecho de autor" o "derechos de los usuarios de obras protegidas por derecho de autor"
} 
En base al estudio de las legislaciones en los países miembros del Mercosur, Díaz Charquero (s.f.) define las siguientes excepciones que caracteriza como« básicas y necesarias» (Díaz Charquero, s.f.:9): copia privada, excepciones para actividades educativas, para investigación, para bibliotecas y prestamos público de obras. Destaca que esta última forma parte de las que corresponden a las bibliotecas, pero las incorpora como una categoría particular basada en que esta práctica se encuentra ampliamente extendida los países del Mercosur, pero sólo prevista como práctica lícita en Paraguay.

Estos límites al derecho de explotación de la obra por parte de los autores son expresados en el Convenio de Berna en el artículo 2 bis, 9 y 10. Es necesario poner énfasis en que las limitaciones refieren siempre a los derechos de explotación, no a los derechos morales.

Los antecedentes normativos de las limitaciones y excepciones pueden rastrearse en la declaración de los DDHH. En su artículo 27 expresa los derechos de acceso a las obra como el de los autores a la protección. En el mismo sentido, el Pacto Internacional de Derechos Económicos, Sociales y Culturales (PIDESC) hace referencia en su artículo 15 a los siguientes derechos: 1) El derecho de toda persona a la protección de los intereses morales y materiales que le corresponda por razón de las producciones científicas, literarias o artísticas de que sea autora; y 2) el derecho de toda persona a tomar parte libremente en la vida cultural de la comunidad, a gozar de las artes y a participar en el progreso científico y en los beneficios que de él resulten. (Díaz Charquero, S.f.: 2)

Estos derechos también fueron incluidos en el Convenio de Berna, en 1974. En su artículo 9, el tratado expresa: en el entendido expresado en el Convenio de Berna y en nuestra ley: « siempre que no atente a la explotación normal de la obra ni cause un perjuicio injustificado a los intereses legítimos del autor». (Convenio de Berna, Art. 9) Por su parte el tratado de la OMPI (1996) en el artículo 10, también sugiere cómo reglamentar las limitaciones y excepciones.

En el Acuerdo sobre los Aspectos de los Derechos de Propiedad Intelectual relacionados con el Comercio (ADPIC), suscrito en 1994, se consagra, en el 
artículo 13 esta "regla de los tres pasos", procedimiento para instrumentar limitaciones y excepciones. ${ }^{3}$

Algunos países han utilizado las limitaciones y excepciones como forma de adaptar su marco normativo a la realidad tecnológica. Ejemplo de esto son los países de la Unión Europea, que incluyeron en sus respectivas normativas las recomendaciones de la OMPI.

Según estudios de la OMPI, la ley uruguaya de derecho de autor no cuenta con limitaciones y excepciones en general, ni tampoco en favor de las bibliotecas. La legislación nacional es «la más desactualizada por ende más restrictiva» (Díaz Charquero, s.f.: 9) de los países del Mercosur.

En lo que respecta a las excepciones y limitaciones para las bibliotecas Díaz Charquero (s.f.) las define como « aquellas que implican la reproducción de obras de su catálogo con fines de preservación, adquisición de ejemplares indisponibles o en préstamo interbibliotecario, digitalización y disponibilización en terminales, préstamo digital (...). Y también otras excepciones que se relacionan con el uso legal de obras huérfanas o traducción de obras con fines de investigación o educación, entre otras». (Díaz Charquero, s.f.:9)

Por su parte, Crewss (2008) afirma que «las excepciones al derecho de autor aplicables a las bibliotecas constituyen una parte importante de las legislaciones sobre el derecho de autor desde 1956» (Crewss, 2008:14) ubicando como primer antecedente a la promulgación realizada por el parlamento británico, que las incluyó en su Ley de Derecho de Autor.

\section{Metodología}

El diseño de investigación corresponde a la teoría fundamentada, desde la perspectiva de este trabajo se entiende como aquella en la que «el investigador produce una explicación general o teoría respecto a un fenómeno, proceso, acción o interacciones que se aplican a un contexto concreto y desde la perspectiva de diversos participantes». (Hernández Sampieri, 2014: 472)

\footnotetext{
${ }^{3}$ Art. 13 del Acuerdo sobre los aspectos de los Derechos de Propiedad Intelectual Relacionados con el Comercio: «Los Miembros circunscribirán las limitaciones o excepciones impuestas a los derechos exclusivos a determinados casos especiales que no atenten contra la explotación normal de la obra ni causen un perjuicio injustificado a los intereses legítimos del titular de los derechos.»
} 
Se han tomado en el transcurso de la investigación aspectos de la Teoría Crítica, no en el sentido estrictamente metodológico sino en relación a su interpelación crítica de la realidad. En tal sentido, la investigación se enmarca en términos generales bajo un punto de vista crítico «que invita al sujeto a un proceso de reflexión y análisis sobre la sociedad en la que se encuentra implicado y la posibilidad de cambio que el mismo es capaz de generar.» (Melero Aguilar, 2012: 344)

\subsection{Definición de las variables}

Las variables definidas en este este trabajo son las siguientes:

a) Expectativas y demandas de los usuarios vinculadas a la reproducción, difusión y disposición del público de las obras bajo derecho de autor de acuerdo a la percepción en las unidades de análisis.

b) Servicios brindados acordes a esas expectativas y demandas, y servicios afectados en función de la ley vigente.

c) Conocimiento y valoración sobre el cumplimiento de la ley vigente por parte de los profesionales de la información.

\subsection{Muestra y selección}

De acuerdo con el diseño general de la investigación, de enfoque cualitativo, se seleccionó una muestra no probabilística de tipo intencional o por conveniencia, la cual «pretende profundizar en aspectos más concretos de la realidad social, a través de la interpretación y análisis de los discursos de los actores sociales, sin perseguir la generalización de los resultados a la población general en términos calculables estadísticamente.» (Palacios y Rubio, 2003: 60)

Durante el desarrollo de selección de la muestra hemos prevalecido la accesibilidad a la información fiable que puede brindar cada unidad de análisis, tendiendo a que se encuentre accesible y proporciones resultados sustanciosos. (Patton, citado por Martínez-Salgado, 2012: 616) También se tuvo en cuenta que los datos fueran pasibles de ser constatados mediante la observación. 


\subsection{Tamaño de la muestra y unidades de análisis}

El tamaño de la muestra fue establecido según el principio de flexibilidad que propone Patton (citado por Martínez Sagado, 2012 :616)

Esta perspectiva se ajustó con precisión a la investigación, ya que se contaba con el conocimiento previo del contexto en el que se iba a intervenir y con la posibilidad de acceder a fuentes de información fiable.

\subsection{Unidades de análisis}

Las unidades de análisis fueron 12 bibliotecas de la ciudad de Montevideo. Una de las principales dificultades en cuanto a la accesibilidad de datos fue el hecho de tener que consultar sobre las prácticas y las percepciones de las demandas de los usuarios en relación al cumplimiento o no de una ley.

Por eso, para lograr un acercamiento a datos fiables fue necesario tomar las siguientes acciones: asegurar el anonimato a los participantes, recurrir a quienes toman las decisiones en las bibliotecas: directores, encargados, contrastar los datos recaudados en las entrevistas con la observación de los servicios difundidos en las web, tener en cuenta que los datos recabados están vinculados directamente a la percepción de los participantes de la muestra, y en ese sentido, brindar la confianza necesaria para contar con datos fiables.

Los requisitos excluyentes para formar parte de la muestra se centraron en que las unidades de información contaran con: servicios a través de las TICS y estar a cargo de un profesional licenciado en Bibliotecología.

\subsection{Herramientas para la recolección de los datos}

En cuanto a las técnicas de recolección de datos se optó por una triangulación, entendiendo ésta como «la utilización de diferentes estrategias y fuentes de información sobre una recogida de datos permite contrastar la información recabada.» (Aguilar y Barroso, 2015: 74)

\subsubsection{Observación}

Se realizó una observación directa de los catálogos y la difusión de los servicios de las unidades de información en la web. Tal observación fue considerada como 
una observación investigativa, en la cual no solo se ve, sino que se intenta observar y estar atento a los detalles, «sucesos, eventos e interacciones.»(Hernández Sampieri, 2014: 398)

\subsubsection{Entrevistas}

\section{Exploratorias y abiertas.}

Se realizaron en primera instancia seis entrevistas exploratorias o «piloto» (Hernández Sampieri, 2014: 403) que acercaron la investigación al tema, proporcionaron la información necesaria para comenzar a indagar a nivel teórico y estructurar las entrevistas conforme fue avanzando el trabajo de campo y suministraron datos para poder incorporar unidades de análisis a la muestra.

\section{Entrevistas semiestructuradas.}

Una vez consolidado el tema y a la luz de los primeros datos, se formuló la hipótesis y se establecieron las variables. Así fue posible diseñar la entrevista de manera tal que respondiera las preguntas vinculadas a las variables.

Fueron realizadas 12 entrevistas de manera semiestructurada con base en las variables planteadas, las mismas fueron conducidas con libertad a fin de poder trasmitir con claridad los conceptos y hacer comprensibles las preguntas formuladas; y sobre todo brindar la confianza necesaria para obtener respuestas veraces aun cuando pudieran evidenciar el incumplimiento de la legislación vigente.

\subsection{Categorización y análisis de los datos}

Teniendo en cuenta el volumen de información que se recopiló en las entrevistas y a través de la observación -algo que es propio de los estudios de corte cualitativo-, se optó por definir categorías y subcategorías. Esto implica un proceso de identificación de unidades temáticas significativas, que dentro de una masa amplia de información aportan los datos en forma organizada y permiten su clasificación para el análisis posterior (Miles y Huberman, citado por Mejía Navarrete, 2011:51). Según Navarrete (2011:51), el análisis está pautado por tres fases interrelacionadas: 1- edición, categorización, codificación, clasificación y 
presentación de datos. 2- análisis descriptivo para elaborar conclusiones empíricas y descriptivas, 3- la interpretación, con el fin de abordar explicaciones

\subsection{Reducción de los datos: categorización y clasificación}

Las categorías fueron conformadas de acuerdo al planteo de D'ancona, según el valor de la variable: «los atributos son las distintas categorías o valores que componen la variable.» (1996:26). Estos valores son proporcionados por lo que Hernández Sampieri (2014: 438) llama «material vinculado a la teoría.»

Las categorías y subcategorías, (ver cuadro 1) respondieron a la repetición de la información en las entrevistas exploratorias. A través del razonamiento inductivo conformamos cada categoría.

Es preciso indicar la diferencia entre la categoría 2 y 3, ya que las subcategorías son similares. En la categoría 2 la finalidad es indagar en las prácticas reales y cotidianas que tienen lugar en las bibliotecas en función de las demandas de los usuarios y las prácticas de los profesionales.

La categoría 3 apunta a conocer cuáles servicios son ofrecidos a los usuarios, mediante la observación en la web de las bibliotecas. Con esto se buscó contrastar la difusión pública de los servicios ofrecidos (difusión que no puede exponer la transgresión a la ley de derecho de autor), y las demandas de usuarios y prácticas reales por parte de los profesionales, vinculadas a la reproducción, difusión y puesta a disposición de la información al público. 
Cuadro 1: Categorías y Subcategorías

\begin{tabular}{|c|c|c|}
\hline Categoría & Sub-categorías & Origen de los datos \\
\hline CATEGORIA 1 & & Entrevista \\
\hline \multirow[t]{4}{*}{$\begin{array}{l}\text { Demandas de los usuarios vinculadas a la } \\
\text { prácticas de reproducción }\end{array}$} & $\begin{array}{l}\text { Utilidad del catálogo } \\
\text { referencial }\end{array}$ & \\
\hline & $\begin{array}{l}\text { Solicitud de servicios a } \\
\text { texto completo }\end{array}$ & \\
\hline & $\begin{array}{l}\text { Solicitud de servicios de } \\
\text { reproducción }\end{array}$ & \\
\hline & $\begin{array}{l}\text { Reproducción por parte } \\
\text { de los usuarios }\end{array}$ & \\
\hline CATEGORÍA 2 & & Entrevista \\
\hline \multirow[t]{4}{*}{ Práctica basadas en la reproducción } & Fotocopiado & \\
\hline & Escaneado & \\
\hline & $\begin{array}{l}\text { Envío documentos o links } \\
\text { por mail }\end{array}$ & \\
\hline & Copia de preservación & \\
\hline CATEGORÍA 3 & & Observación \\
\hline \multirow[t]{3}{*}{$\begin{array}{l}\text { Servicios ofrecidos y promocionados } \\
\text { vinculados a la reproducción }\end{array}$} & Fotocopiado & \\
\hline & Escaneado & \\
\hline & $\begin{array}{l}\text { Envío documentos o links } \\
\text { por mail }\end{array}$ & \\
\hline CATEGORÍA 4 & & Entrevista \\
\hline \multirow[t]{3}{*}{$\begin{array}{l}\text { Conocimiento de la ley y valoración sobre } \\
\text { su aplicación }\end{array}$} & $\begin{array}{l}\text { Medidas de protección en } \\
\text { cuanto a la exposición }\end{array}$ & \\
\hline & $\begin{array}{l}\text { Desconocimiento sobre } \\
\text { la ley }\end{array}$ & \\
\hline & $\begin{array}{l}\text { Prohibición de } \\
\text { reproducción }\end{array}$ & \\
\hline
\end{tabular}

\section{Resumen del análisis descriptivo e interpretativo de los resultados}

\subsection{Categoría 1: Demandas de los usuarios vinculadas a las prácticas de reproducción}

\section{Subcategorías}

\section{Utilidad del catálogo referencial}

La totalidad de los entrevistados cuenta con un catálogo automatizado y referencial ya sea en la web o en Intranet. Sobre las particularidades en el uso del catálogo de acceso público en línea se plantearon diferencias entre las unidades de información estudiadas: 
El catálogo referencial es utilizado por los bibliotecólogos/as o funcionarios/as quienes hacen las búsquedas para ubicar mediante la signatura topográfica el libro en soporte papel. No es por tanto de uso frecuente por parte de los usuarios. Destacamos la identificación del catálogo referencial en la web con el soporte físico a pesar de que son herramientas que pueden incorporar documentos digitales. En otras palabras, tecnológicamente es posible no restringir el catálogo a la colección en soporte físico se podría además contener documentación a texto completo. De esto se puede inferir que se trata de una herramienta que actualmente está subutilizada.

\section{Solicitud de servicios a texto completo}

De acuerdo a los datos relevados, el acceso a texto completo y remoto es una demanda recurrente y claramente expresada. Ello implica la obtención de la obra en soporte digital a través de su descarga de Internet o su reproducción mediante escaneo o fotografía, de manera tal que quede accesible mediante su incorporación al catálogo en línea o su envío por correo electrónico.

Esto no debe suponer necesariamente la reproducción ilícita o la omisión a la ley de derechos de autor por parte de los profesionales. Pero sí parece ser un indicador de la necesidad de los usuarios en acceder a documentos en forma integral y no a un dato referencial. Desde la percepción del profesional, parecería que los usuarios desconocen la normativa y solicitan ese servicio por considerarlo algo natural en una biblioteca. Resulta esperable que la demanda de los usuarios radique en el acceso irrestricto a los contenidos, demanda que naturalmente trasladan a la biblioteca.

Las TICS han ofrecido a los usuarios herramientas de búsqueda y acceso a todo tipo de documentos y han habilitado además que puedan ser reproducidos y compartidos Entonces, ¿por qué no habría de hacerlo una biblioteca?

Los hábitos de los usuarios se ven modificados a raíz de los cambios tecnológicos, como ocurre con el acceso inmediato a la información en redes. Esto redunda, entre otras cosas, a que y se establezcan expectativas y costumbres vinculadas a acceder al texto completo sin restricciones.

La edad parece ser un factor que influye en este tipo de hábitos. Los más jóvenes tienen una clara demanda de servicios y accesos remotos a textos completos y no reparan en la existencia de normativas que lo limiten. 
La expectativa natural es acceder en el menor tiempo posible a los contenidos en forma integral y remota. Podemos decir que hay una percepción naturalizada de la reproducción y la libertad de acceso a los contenidos por parte de los usuarios, la cual no radica en el desconocimiento de la normativa vigente (aunque probablemente la desconozcan) sino que parte de una concepción sobre el acceso a los contenidos propia de la Sociedad de la Información.

En base a lo dicho, no parece que los usuarios observen las restricciones, y en este marco la ley de derecho de autor implica para las bibliotecas un conflicto de tres frentes: lo que sería una buena práctica de su cometido esencial, brindar acceso a la información; atender las demandas actuales de los usuarios y cumplir con la ley.

\section{Solicitud de servicios de reproducción: escaneado y fotocopias}

La amplia mayoría de los entrevistados plantearon la solicitud expresa de los usuarios en cuanto a la demanda de reproducción mediante acceso remoto, fotocopiado de los documentos en soporte papel o escaneado y envío por correo electrónico de los documentos en soporte digital.

Las bibliotecas acceden a dar los servicios de reproducción solicitados con algunas limitaciones, como veremos en la categoría 4.

El caso de la demanda de reproducción por escaneado y fotocopiado parece ser muy similar al de la demanda de acceso a texto completo. Se trata de solicitudes percibidas por los usuarios como naturales en una biblioteca.

El escaneado se asimila cada vez más al fotocopiado. Es una herramienta que se ha difundido ampliamente y brinda muchas ventajas: la posibilidad de acceso remoto por múltiples usuarios, la facilidad de difusión y las ventajas de conservación respecto a copias en papel y respaldo de información en espacios reducidos. También es ampliamente solicitado por los usuarios.

\section{Reproducción por parte de los usuarios}

El avance en cuanto a las aplicaciones (app) en los celulares también trajo aparejada la posible reproducción a través de la fotografía por parte de quien quiera hacerlo. Se observa una aparente práctica extendida en este aspecto $\mathrm{y}$, tal como se vio en la categoría vinculada a la demanda, pareciera que los usuarios no perciben la reproducción como un hecho ilícito o como una actividad que requiera algún tipo de autorización: 
En algunos servicios se pide expresamente "no sacar fotos", pero en los hechos es un control que escapa a las posibilidades y funciones de las bibliotecas. El control se intenta realizar a través de limitaciones realistas, permitiendo tomar fotografía de una parte del documento. En esta subcategoría analítica, también se encontró mención a la edad como un factor diferencial. Parecería que en el caso de los jóvenes la naturalización de la reproducción es más clara, lo cual puede ser algo esperado a raíz del vínculo entre los millennians y el uso de las TICS.

Percepción de las necesidades de los usuarios por parte de los/as Bibliotecólogos/as:

En las entrevistas se hace mención a que las necesidades de los usuarios que tienen un gran manejo de las TICS están pautadas por esa habilidad. Para estos usuarios, el deseo y la demanda concreta refieren usualmente al acceso a los contenidos en forma inmediata en soporte digital.

También se hace mención a la tipología de usuarios que recién comienzan a desarrollar competencias informáticas, entendidas como " el conjunto de conocimientos, habilidades, disposiciones y conductas que capacitan a los individuos para saber cómo funcionan las TICS; para qué sirven; y cómo se pueden utilizar para conseguir objetivos específicos." (Comisión Mixta CRUETIC-REBIUM, 2009:7). Estos usuarios generan expectativas respecto a sus posibilidades, que pasan a ser irreales cuando enfrentadas a las posibilidades legales e incluso tecnológicas.

Podríamos interpretar que los usuarios se dividen en dos grandes grupos: los que recurren a las TICS en forma naturalizada y con la necesidad y expectativa de ingresar a los contenidos digitales; y los que recién comienzan a desarrollar las competencias informáticas y alimentan expectativas que no vinculan con normativas legales que puedan limitarlas.

Se percibe incluso ya en esta categoría, un cuestionamiento ético en cuanto al cumplimiento de la función profesional en cuanto a las necesidades percibidas.

La existencia de las herramientas para la reproducción tanto en soporte papel (fotocopias) como en soporte digital (escaneado), y la naturalización por parte de los usuarios de libertad de acceso en contraposición con la ley, colocan al profesional de la información en un lugar como mínimo de cuestionamiento sobre sus prácticas. 


\subsection{Categoría 2: Prácticas basadas en la reproducción}

Es preciso recordar que esta categoría aborda datos sensibles debido a que los entrevistados deben reconocer que cometen un acto ilícito.

\section{Subcategorías}

\section{Fotocopiado y Escaneado}

En su amplia mayoría los servicios consultados escanean y fotocopian documentos. El fotocopiado se encuentra naturalizado y es un servicio habitual y cotidiano. El escaneado si bien no es reconocido como un servicio explícito es habitual y realizado ante la demanda de los usuarios.

Asimismo, en este punto se pudieron observar algunas creencias sobre limitaciones a las prácticas que tendrían como finalidad ajustarse a la normativa, pero que de hecho son ilícitas (lo cual veremos específicamente en la categoría 4). Por ejemplo, se planea como limitante únicamente la reproducción de textos que produce la misma institución.

Las instituciones no son propietarias de derechos, el propietario de esos derechos es el autor, son derechos morales y no pueden ser cedidos. En el estricto cumplimiento de la ley debería existir una autorización explícita para que la institución realice esas prácticas. No obstante, suele considerarse que lo que es producido en el marco de una institución tiene de por sí los derechos adquiridos, pero se trata de un concepto equivocado. Otro ejemplo de creencias erróneas es concebir la reproducción parcial de una obra o de imágenes como una limitación para respetar la ley.

Queda claro que en todos los casos existe algún grado de percepción de que se está infringiendo la norma, ya que los testimonios dan cuenta de intentos de restricción o de no difusión de la práctica. El punto de inflexión es que nuestra legislación no provee prácticamente excepción alguna y por tanto la restricción debería ser total.

\section{Envío de documentos o enlaces por correo electrónico}

El envío por correo electrónico de documentos o enlaces de origen heterogéneo es una práctica llevada a cabo por todas las unidades entrevistadas.

En algunas unidades recurren al Portal Timbó, que proporciona la seguridad de documentos con libre acceso. En otras se reproducen los documentos, incluso mediante fotografías, y se envían a los usuarios. También se estila enviar 
documentos y enlaces tanto de suscripciones pagas o gratuitas como de Internet en general.

\section{Copia de preservación}

En la media, los servicios realizan algún tipo de copia con finalidades de preservación y en algún caso de acceso por motivos económicos. Otras lo hacen en casos puntuales.

\subsection{Categoría 3: Servicios ofrecidos públicamente y promocionados vinculados a la reproducción}

Esta categoría se basó en la observación de los servicios promocionados mediante la información contenida en la web.

La intención fue comparar los servicios vinculados a la reproducción que efectivamente se brindan y aquellos que se informan y promocionan por parte de la biblioteca. Prácticamente ningún servicio de información hace promoción sobre la posibilidad de reproducir o enviar documentos a texto completo, más allá de los contratados. En consecuencia son servicios que se brindan pero no se difunden como tales. No son ofrecidos como un servicio explícito.

\subsection{Categoría 4: Conocimiento de la ley y valoración sobre su aplicación}

\section{Subcategorías}

\section{Medidas de protección o reducción de exposición}

Todos los entrevistados plantean la adopción de algún tipo de medida en el intento por limitar infracciones a la ley.

En algunos casos las medidas son formales y expresas, escritas y/o aplicadas por todo el personal como norma específica ante el tema. Algunos optan por aplicar la "regla de los tres pasos", aprobada en el Convenio de Berna, la cual tiene como cometido restringir los diferentes tipos de "uso justo" para aquellos países que incorporaron limitaciones y excepciones en sus leyes de derecho de autor y conexos.

En otros casos no se encuentran formalmente escritas pero el personal de la biblioteca las aplica. Fotocopian partes de capítulos, o no se escanean documentos físicos, o realizan excepciones mediante consentimiento de los jerarquías superiores. Es pertinente destacar las excepciones por solicitud escrita 
de superiores: varios entrevistados plantearon la dificultad extra que no son sólo los usuarios directos quienes naturalizan la reproducción, sino que también realizan ese tipo de demandas funcionarios de mayor jerarquía y tomadores de decisiones.

Otras bibliotecas, por su parte, no cuentan con restricciones formales pero sí informales y de carácter general, que apuntan a evitar la exposición y restringir el servicio. En otros casos es clara la visualización de la ley de derecho de autor con la limitante clara y concreta sin posibilidad de realizar restricciones. En otros casos se cumple con la ley al tomarse consciencia de las restricciones que impone. Tal como se vio en categorías anteriores, es clara la vocación por atender las demandas y necesidades del usuario. Tanto las políticas formales como las informales están orientadas a generar la comprensión por parte de los usuarios y a implementar restricciones que no afecten demasiado el acceso a la información.

\section{Autovaloración sobre el conocimiento de la ley y la aplicación en cada servicio}

Un insumo considerado clave para la investigación es la opinión de los profesionales a cargo de las bibliotecas respecto a su propio conocimiento sobre la ley de derecho de autor y su aplicación en los servicios que prestan.

En general, hay una autovaloración positiva sobre el conocimiento de la ley y un reconocimiento de las dificultades que surgen a partir de la decisión de aplicarla o no en sus servicios. Queda en evidencia, a partir de las respuestas, el rol que ocupan como mediadores entre la ley y las demandas de los usuarios de acceder a ella. En ese escenario, deben intentar cumplir con su rol y a la vez evitar quedar expuestos ejecutando prácticas ilícitas.

\section{Prohibición de reproducción (C4-S3)}

Respecto a la prohibición expresa de reproducción por parte de los usuarios, la gran mayoría de los profesionales entrevistados, a excepción de dos, no prohíben la reproducción. Incluso se observó cómo adoptan medidas para reducir la exposición de la institución al permitir esas acciones por parte de los usuarios: no prohíben reproducir a los usuarios, por ej. ellos sacan su propias fotocopias.

Vale la pena destacar la autopercepción que tienen estos profesionales sobre sobre su función de facilitar el acceso a la información, función a la que dan un valor que trasciende incluso el fundamento ético de atenerse a una norma. Como 
queda plasmado en el hecho de que muchas veces están dispuestos ya bien a incumplir la ley o intentar formas de poder llevar a cabo su rol en una realidad normativa como la actual.

\subsection{Consideraciones finales sobre el análisis de los datos:}

El conflicto entre las necesidades de los usuarios y el cumplimento de la ley también parece ser evidente.

Luego de indagar en la categoría 4 se pudo observar que las acciones de reproducción por parte de los profesionales de la información no se deben a un desconocimiento sobre la ley sino de una real imposibilidad de cumplirla.

Un rol que emerge como necesario ante este escenario es el de asesor en prácticas institucionales. Dicho de otro modo, la interpretación de los datos obtenidos sugiere que las acciones para satisfacer las necesidades del público están directamente reñidas con el cumplimiento de la ley vigente.

El presente análisis interpretativo concuerda con la situación en Argentina, dónde el marco normativo en materia de limitaciones y excepciones en favor de las bibliotecas es similar al de Uruguay. Dice Pelaya (2015): “ ...es incomprensible que la legislación argentina no contemple un régimen de excepciones para que dichas instituciones puedan cumplir con su misión en un marco de legalidad" (Pelaya, 2015: 121).

\section{Conclusiones}

Las conclusiones abordadas no pretenden ser definitivas. Surgieron en el proceso de investigación e intentan aportar una aproximación a las particularidades que implica la vigencia de la Ley de Derecho de Autor en Uruguay en el marco de la Sociedad de la Información.

El enfoque que tiene este trabajo parte de una perspectiva crítica, por lo cual se abordó el objeto a estudiar desde un punto de vista que cuestiona la normativa vigente en función de la realidad en la que se enmarca y a la luz de las prácticas que se desarrollan a diario en las bibliotecas.

\subsection{De las prácticas y el cumplimiento de la ley}


Es preciso recordar que el presente trabajo no contempla un estudio de las necesidades y demandas de los usuarios, sino que se basa en la percepción que los profesionales de la información tienen respecto a las mismas.

Es en base a esa percepción que estos profesionales definen sus prácticas y los servicios ofrecen, los cuales incluyen en muchos casos «la impresión, fijación, reproducción, distribución, comunicación o puesta a disposición del público de una obra sin consentimiento del autor.» (Ley $\mathrm{N}^{\circ} 17.616$, modificación del numeral $1^{\circ}$ del literal B) de la Ley $\mathrm{N}^{\circ}$ 9.739).

Tales prácticas constituyen actos susceptibles de sanciones en caso de cumplimiento estricto de la ley, pero a la vez involucran la función principal de las bibliotecas, que es dar acceso a la información. Esta contradicción obliga a los profesionales a actuar fuera del marco legal cuando intentan cumplir en forma cabal su función, ya que no es posible, bajo el paradigma social de la Ciencia de la Información, respetar una norma reñida con los intereses de los usuarios, que además prohíbe el aprovechamiento de todos los recursos que ofrecen las TICS.

\subsection{De las limitaciones y excepciones}

Ley 9.739 de Derecho de Autor no incluye limitaciones y excepciones para las bibliotecas, como tampoco ningún inciso que pueda operar en favor de las prácticas y servicios mencionados.

Vale recordar que es una ley cuya redacción data del año 1937 y que no fue objeto de modificaciones sustanciales pese a los cambios radicales que ha sufrido el escenario tecnológico. El artículo 45 de la ley, es destinado a actos lícitos, que son inoperantes a los efectos de brindar flexibilidad para el acceso a la información. No contempla posibles limitaciones al derecho exclusivo del autor sobre la obra, desconociendo las recomendaciones de la OMPI, que han sido claras en el sentido de flexibilizar las legislaciones nacionales e incorporar limitaciones y excepciones de diferente naturaleza.

\subsection{De los profesionales de la información}

Ante el escenario actual, los/as bibliotecólogos/as no desconocen que brindan servicios no amparados por la ley. Esta situación los enfrenta a un dilema ético: cumplir su función esencial o violar la normativa vigente. 
Ante esa situación, los profesionales intentan minimizar los riesgos que conlleva infringir la ley mediante acciones destinadas a disminuir su grado de exposición, como limitar la cantidad de reproducciones y la modalidad digital y/o solicitar autorización a sus superiores para contar con un mayor respaldo.

Resulta llamativo que en algunos casos recurran a la regla de los tres pasos propuesta por la OMPI, lo que significa optan por seguir una recomendación internacional antes que ésta esté contemplada en el sistema jurídico de su país.

\subsection{De la ley}

El caso de la Ley 9.739 del año 1937, la cual fue objeto de modificaciones puntuales desde su promulgación, parece ser un ejemplo de la imposibilidad del sistema jurídico de acompasar las necesidades de las sociedades.

Eso se evidencia tanto en las limitaciones que la ley vigente supone para los servicios en las bibliotecas, como en el naturalizado no cumplimiento de la ley en los ámbitos privados.

En el caso de las bibliotecas, son instituciones que difunden y brindan servicios, y cuyos profesionales actúan conforme a un código de ética, razón por la cual se encuentran expuestas a ser intimadas por violación a la Ley de Derecho de Autor. En la Ciencia de la Información no es nueva la discusión sobre el acceso a los contenidos en Internet y sus complejidades en cuanto a cantidad, calidad y pertinencia de recuperación y control.

Desde el área del Derecho tampoco es un tema saldado, aunque esto quizás tenga relación con que el orden jurídico no es un todo coherente y presenta ambigüedades. (Contreras, 2013)

Se trata de un tema complejo que no se dirime fácilmente, es una discusión abierta en la que intervienen varios actores con intereses y enfoques diferentes. Prueba de ello es que la única modificación fue realizada en diciembre del 2019 (terminando el ejercicio parlamentario ante un cambio de gobierno) extendiendo los derechos patrimoniales de los autores de 50 a 70 años. Este acto jurídico tiene una implicancia directa en el acceso a algunas obras que pasaban a dominio público, por ejemplo la de Felisberto Hernandez y es una modificación sustancial y simbólica. Conforme cambia la realidad hacia mayores posibilidades de acceso, opera con mayor firmeza la lógica de control y la protección hacia los autores, sin 
que se logre que medien en esa situación mecanismos que trasciendan el derecho punitivo y resuelvan la posibilidad de brindar acceso a obras culturales.

Entre la tentación de las posiciones liberales y la protección desmedida de un sector como los autores, contamos con una ley que no ofrece el equilibrio necesario en una sociedad signada por los recursos digitales y el acceso masivo a la información.

Desde la Ciencia de la Información es necesaria una acción imperante que implique una lectura de la realidad crítica y abierta que genere la adaptación de la norma con el fin de legitimar su aplicación y garantizar su cumplimiento (sin caer en el terreno de lo estrictamente punitivo).

Esto puede realizarse a través de las limitaciones y las excepciones de la Ley de Derecho de Autor en favor de las bibliotecas, tal como recomienda la OMPI.

Es al menos curioso que el sistema normativo uruguayo mantenga la vigencia de una ley que no es legitimada en la realidad. Más allá de la eventual solución que conllevaría la implementación de las limitaciones y excepciones de la ley en favor de las bibliotecas, surge una pregunta a en referencia a las sanciones previstas: ¿Respecto a la problemática en torno al derecho de autor, puede considerarse al derecho punitivo como un horizonte posible en la Sociedad de la Información?

Es una pregunta que establece un diálogo posible entre la Ciencia de la Información y el Derecho, pero a la vez interpela desde la práctica el modo de intervenir en la realidad a todos los actores que participan en este tema.

\section{Referencias bibliográficas}

BALSA, M., BUGALLO, B. (2011). Industrias Creativas y Propiedad intelectual. Montevideo : Dicrea.

BUGALlO MONTAÑO, B. (2006). Propiedad intelectual. Montevideo: Fondo de Cultura Universitaria.

CONTRERAS, S. (2013). Derecho positivo y derecho natural: una reflexión desde el iusnaturalismo sobre la necesidad y naturaleza de la determinación. Kriterion: Revista de Filosofia, 54(127), 43-61 
COMISIÓN MIXTA CRUE-TIC; REBIUN (2009). Competencias informáticas e informacionales en los estudios de grado. [documento en línea]. Recuperado de:

http://213.32.37.214/xmlui/bitstream/handle/20.500.11967/49/documento $\underline{\text { competencias_informaticas-2009.pdf?sequence }=1 \& \text { isAllowed }=y}$

CONVENIO, D. B. P. L. P., \& DE LAS OBRAS LITERARIAS, Y. A. Del 9 de Septiembre de 1886, revisado en París el 24 de Julio de 1971 por Instrumento de 2 de Julio de 1973. Recuperado de: https://biblioteca.ua.es/es/propiedadintelectual/documentos/legislacion/convenio-de-berna.pdf

CREWS, K. (2008). Estudio sobre las limitaciones y excepciones al derecho de autor en beneficio de la las bibliotecas y archivos. Ginebra: OMPI. Recuperado de: https://www.wipo.int/meetings/es/doc_details.jsp?doc_id=109192

CREWS, K. (2017). Resumen del estudio sobre las limitaciones y excepciones al derecho de autor en beneficio de la las bibliotecas y archivos. Versión actualizada. Ginebra: OMPI. Recuperado de: https://www.wipo.int/edocs/mdocs/copyright/es/sccr_35/sccr_35_6.pdf

de los Derechos Humanos, D. U. (2003). Declaración Universal de los Derechos humanos. Tomado de http://www. aprodeh. org. pe.

de la Federación, D. O. (1994). Acuerdo sobre los aspectos de los Derechos de Propiedad Intelectual Relacionados con el Comercio. Recuperado de: http://www.propiedadintelectual.udelar.edu.uy/wpcontent/uploads/2016/09/AADPIC-.pdf

DIAZ CHARQUERO, P. (s.d.). Situación de las excepciones y limitaciones a los Derechos de Autor en los países del MERCOSUR desde la perspectiva del acceso al conocimiento y a la cultura. [documento en línea]. Montevideo, Uruguay. Recuperado de: http://bib.fcien.edu.uy/jbdu2015/wpcontent/uploads/2015/05/D\%C3\%ADaz_Situaci\%C3\%B3n-de-las- 
excepciones-y-limitaciones-a-los-Derechos-de-Autor-en-lospa\%C3\%ADses-del-MERCOSUR.pdf

GUIMARÃES, J.A., SANTOS, J.C. y FERNÁNDEZ-MOLINA, C. (2018). A Informação como assunto no direito e na ciência da informação: um estudo comparativo a partir da realidade brasileira. Scire, 24:1 (ene-jun), 45-54

HERNANDEZ SAMPIERI, R., FERNANDEZ COLLADO, C., BAPTISTA LUCIO, P. (2014). Metodología de la investigación. México: McGraw Hill.

LEY 9739. Ley de Derecho de Autor. Poder Legislativo Montevideo, Uruguay, 17 de diciembre de 1937.

LEY $N^{\circ}$ 17616. Ley de Derecho de Autor y Derechos Conexos. Poder Legislativo Montevideo, Uruguay, 10 de enero de 2003. Recuperable

MARTINEZ - SALGADO, C. (2012). El muestreo en investigación cualitativa: principios básicos y algunas controversias. Ciência \& Saúde Coletiva, 17, 613-619. Recuperado de:

https://www.scielosp.org/pdf/csc/2012.v17n3/613-619/es

MELERO AGUILAR, N. (2012). El paradigma crítico y los aportes de la investigación acción participativa en la transformación de la realidad: un análisis desde las ciencias sociales. Cuestiones pedagógicas, 21, 339-355. Recuperado de: https://idus.us.es/xmlui/bitstream/handle/11441/12861/file_1.pdf?sequenc $\underline{e=1 \& \text { is Allowed }=y}$

NAVARRETE, M. (2011). Problemas centrales del análisis de datos cualitativos. Revista latinoamericana de metodología de la investigación social, (1), 47-60.

OMPI, T. D. L. O. (2005). sobre Derecho de Autor (WCT)(1996). Ginebra, Suiza, Publicación, (226). 
Parte, I. I. Normas relativas a la existencia, alcance y ejercicio de los derechos de propiedadintelectual. Recupeardo de:

https://www.wto.org/spanish/docs_s/legal_s/27-trips_04_s.htm

PELAYA, L. (2015). Excepciones a favor de las bibliotecas en la legislación de derechos de autor - copyright en Estados Unidos, España, Chile y Argentina (tesis de Maestría). Facultad Latinoamericana de Ciencias Sociales (FLACSO). Argentina. 